\title{
石墨烯装载不同含量钴锌铁氧体及其电磁行为对比
}

\author{
马志军, 莽昌烨, 赵海涛, 关智浩, 程亮 \\ (辽宁工程技术大学 矿业学院, 阜新 123000)
}

摘 要: 以天然鳞片石墨为原料制备氧化石墨 $(\mathrm{GO})$, 应用水热法制备钴锌铁氧体 $\left(\mathrm{Co}_{0.5} \mathrm{Zn}_{0.5} \mathrm{Fe}_{2} \mathrm{O}_{4}\right)$, 并将两者制备成 石墨烯 $(\mathrm{rGO}) / \mathrm{Co}_{0.5} \mathrm{Zn}_{0.5} \mathrm{Fe}_{2} \mathrm{O}_{4}$ 复合材料。采用 $\mathrm{X}$ 射线衍射 $(\mathrm{XRD})$ 、拉曼光谱 (Raman)、红外光谱(FT-IR)研究 $\mathrm{rGO} / \mathrm{Co}_{0.5} \mathrm{Zn}_{0.5} \mathrm{Fe}_{2} \mathrm{O}_{4}$ 的结构; 应用透射电子显微镜 (TEM) 和矢量网络分析仪 (VNA) 研究不同复合比例对 $\mathrm{rGO} / \mathrm{Co}_{0.5} \mathrm{Zn}_{0.5} \mathrm{Fe}_{2} \mathrm{O}_{4}$ 复合材料形貌、电磁损耗特性、德拜弛豫模型及电磁响应行为的影响。结果表明: 复合反应后 的 GO 在 XRD 图谱中主衍射峰由 $2 \theta=9.74^{\circ}$ 变化为 $2 \theta=24.15^{\circ}$, 且红外光谱图中显示含氧官能团消失, 均说明 $\mathrm{GO}$ 成 功还原为 $\mathrm{rGO}$ 。透射电子显微镜图中可以看到 $\mathrm{Co}_{0.5} \mathrm{Zn}_{0.5} \mathrm{Fe}_{2} \mathrm{O}_{4}$ 嵌布在 $\mathrm{rGO}$ 上。复合反应过程中, 当钴锌铁氧体的 含量增大, 分散性逐渐减弱。 $\mathrm{Co}_{0.5} \mathrm{Zn}_{0.5} \mathrm{Fe}_{2} \mathrm{O}_{4}$ 与 $\mathrm{GO}$ 质量比为 $2: 1$ 时制备的 $\mathrm{rGO} / \mathrm{Co}_{0.5} \mathrm{Zn}_{0.5} \mathrm{Fe}_{2} \mathrm{O}_{4}$ 复合材料的吸波性 能最佳，在 $15.11 \mathrm{GHz}$ 处反射率达到最小值-36.89 dB，有效吸波频带宽为 3.74 。

关 键 词: 石墨烯; 复合材料; 钴锌铁氧体; 电磁行为; 氧化石墨烯

中图分类号: TM277; TQ127 文献标识码: A

\section{Comparison of Electromagnetism Behavior of Different Content Cobalt-zinc Ferrite Loaded with Graphene}

\author{
MA Zhi-Jun, MANG Chang-Ye, ZHAO Hai-Tao, GUAN Zhi-Hao, CHENG Liang \\ (College of Mining, Liaoning Technical University, Fuxin 123000, China)
}

\begin{abstract}
A kind of graphene composite $\left(\mathrm{rGO} \mathrm{Co}_{0.5} \mathrm{Zn}_{0.5} \mathrm{Fe}_{2} \mathrm{O}_{4}\right)$ was synthesized with the graphene oxide (GO) prepared from natural flake graphite and cobalt-zinc ferrite $\left(\mathrm{Co}_{0.5} \mathrm{Zn}_{0.5} \mathrm{Fe}_{2} \mathrm{O}_{4}\right)$ manufactured by the hydrothermal method. The structure of composites was characterized by X-ray diffraction (XRD), Raman spectrometer (Raman) and the Fourier-transform infrared spectroscopy (FT-IR). Morphology, electromagnetic loss properties, Debye relaxation, and microwave absorbing properties of $\mathrm{rGO} / \mathrm{Co}_{0.5} \mathrm{Zn}_{0.5} \mathrm{Fe}_{2} \mathrm{O}_{4}$ were investigated by transmission electron microscope (TEM) and vector network analyser (VNA). Sharp peak of graphene oxide was changed from $2 \theta=9.74^{\circ}$ to $24.15^{\circ}$ in the XRD patterns and the oxygen functional group disappeared after the composite reaction, which demonstrated that GO was successfully reduced to rGO. The graphene was embedded with cobalt-zinc ferrite, observed by transmission electron microscope, with its dispersion worse with the loaded $\mathrm{Co}_{0.5} \mathrm{Zn}_{0.5} \mathrm{Fe}_{2} \mathrm{O}_{4}$ increasing. Absorbing property of $\mathrm{rGO} / \mathrm{Co}_{0.5} \mathrm{Zn}_{0.5} \mathrm{Fe}_{2} \mathrm{O}_{4}$ composite prepared with $w\left(\mathrm{Co}_{0.5} \mathrm{Zn}_{0.5} \mathrm{Fe}_{2} \mathrm{O}_{4}\right): w(\mathrm{GO})=2: 1$ is the best with the minimum reflectivity of $-36.89 \mathrm{~dB}$ at $15.11 \mathrm{GHz}$ and the effective absorption frequency bandwidth of 3.74.
\end{abstract}

Key words: graphene; composite materials; cobalt-zinc ferrite; electromagnetic behavior; graphene oxide 
随着现代技术的快速发展，电磁干扰问题日益 凸显，尤其是在通信、军事、民用雷达等应用领域 ${ }^{[1]}$ 。 因此, 电磁波吸收材料成为广泛关注的热点。铁氧 体可以有效地减少电磁波的反射，避免在高频段时 的表面效应，一直以来都是吸波材料领域研究重点 之一 ${ }^{[2]}$ 。Hajalilou 等 ${ }^{[3]}$ 分别以 $\mathrm{MnO} 、 \mathrm{MnO}_{2}$ 为原材料, 并通过控制球磨时间合成了 $\mathrm{Mn}_{0.5} \mathrm{Zn}_{0.5} \mathrm{Fe}_{2} \mathrm{O}_{4}$ 。比较了产 物的微观结构和磁性能, 发现退火后样品的磁化强 度为 $37 \mathrm{emu} / \mathrm{g}$ 。张小川等 ${ }^{[4]}$ 以柠檬酸为络合剂通过 溶胶一凝胶法制备锰锌铁氧体粉末, 得到的粉体的 饱和磁化强度达到 $46.8 \mathrm{emu} / \mathrm{g}$ 。赵海涛等 ${ }^{[5]}$ 采用多 元醇法制备了单分散的 $\mathrm{ZnFe}_{2} \mathrm{O}_{4}$ 纳米颗粒, 发现所 制备的样品尺寸均一, 室温下的饱和磁化强度为 $18.10 \mathrm{Am}^{2} / \mathrm{kg}$, 剩磁较小, 表现出亚铁磁性。铁氧体 虽然具备较好的吸波性能, 但也存在着很多不足, 例如铁氧体的密度较大、吸波频段较窄而且单位体 积 中存储的磁能较低, 饱和磁化强度也较低, 限 制了它在大功率领域的应用。理想的吸波材料应同 时 具有密度低、厚度薄、吸波能力强和吸波频带 宽等特点 ${ }^{[6-8]}$ 。

石墨烯是在六方晶格中形成的二维单层碳原子 层, 由于其在催化、传感器和储能器件等方面的潜 在应用 ${ }^{[9-13]}$, 受到广泛关注。石墨烯在电子、机械和 热性能方面展现出优异的性能 ${ }^{[14-19]}$, 使石墨烯复合 材料受到众多学者的关注。另外, 石墨烯具有“薄、 轻、宽、强”的特点, 性能上可以很好地与铁氧体进 行互补。在吸波材料领域中, 石墨烯复合铁氧体材 料既可以改善材料自身性能, 还可以增强电磁波吸 收能力, 并拓宽吸波频带。Lian 等 ${ }^{[20]}$ 采用气液界面 反应法制备 $\mathrm{Fe}_{3} \mathrm{O}_{4}$-石墨烯纳米复合材料, 并对其电 化学性能进行了探究。 $\mathrm{Li}$ 等 ${ }^{[21]}$ 通过水热法合成了 $\mathrm{CoFe}_{2} \mathrm{O}_{4}$-石墨烯复合材料并研究了其电磁波吸收能 力。Zong 等 ${ }^{[22]}$ 通过水热法制备了不同比例的还原氧 化石墨烯/ $\mathrm{CoFe}_{2} \mathrm{O}_{4}$ 复合材料, 研究表明以 $\mathrm{N}_{2} \mathrm{H}_{4} /$ $\mathrm{NaBH}_{4}$ 为还原剂制备的还原氧化石墨烯 $/ \mathrm{CoFe}_{2} \mathrm{O}_{4}$ 复 合材料在厚度为 $2.3 \mathrm{~mm}$ 、频率为 $12.4 \mathrm{GHz}$ 下达到 最大反射损耗-47.9 dB，低于有效吸波频带 $\left(R_{\mathrm{L}}<\right.$ $-10 \mathrm{~dB})$ 宽为 $5 \mathrm{GHz}$ 。Dan 等 ${ }^{[23]}$ 采用行星球磨法制备 了 $\mathrm{FePCB}$ /石墨烯复合材料, 结果表明片状 $\mathrm{FePCB} /$ 石墨烯复合吸波材料在吸波体厚度为 $2 \mathrm{~mm}$ 、频率为 $12.6 \mathrm{GHz}$ 下达到最大反射损耗 $-45.3 \mathrm{~dB}$, 低于有效 吸波频带 $\left(R_{\mathrm{L}}<-10 \mathrm{~dB}\right)$ 宽为 $5.4 \mathrm{GHz}$ 。因此, 以石墨 烯为基体制备铁氧体/石墨烯复合材料在吸波材料 领域有广阔的发展前景 ${ }^{[24]}$ 。本工作采用水热晶化法 将 $\mathrm{Co}_{0.5} \mathrm{Zn}_{0.5} \mathrm{Fe}_{2} \mathrm{O}_{4}$ 与 $\mathrm{rGO}$ 进行复合制备 $\mathrm{rGO} /$
$\mathrm{Co}_{0.5} \mathrm{Zn}_{0.5} \mathrm{Fe}_{2} \mathrm{O}_{4}$ 复合材料, 并通过改变复合材料中 两种物质的比例, 探究其最佳电磁波吸收性能。

\section{1 实验方法}

\section{1 试剂与仪器}

实验药品: 天然鳞片石墨 $($ 粒度 $180 \mu \mathrm{m}$, 含碳 99.5\%，青岛富润达石墨有限公司); 浓硫酸 $\left(\mathrm{H}_{2} \mathrm{SO}_{4}\right.$, 纯度 $>98 \%$, 青海化工科贸试剂厂); 浓盐酸 $(\mathrm{HCl}$, 纯 度 $>36 \%$, 广州化学试剂厂); 高锰酸钾 $\left(\mathrm{KMnO}_{4}, \mathrm{AR}\right.$, 天津化学试剂一厂); 过氧化氢 $\left(\mathrm{H}_{2} \mathrm{O}_{2}, 30 \%\right.$, 天津大 茂化学试剂厂); 硝酸锌 $\left(\mathrm{Zn}\left(\mathrm{NO}_{3}\right)_{2} \cdot 6 \mathrm{H}_{2} \mathrm{O}\right)$; 硝酸铁 $\left(\mathrm{Fe}\left(\mathrm{NO}_{3}\right)_{3} \cdot 9 \mathrm{H}_{2} \mathrm{O}\right)$; 硝酸钴 $\left(\mathrm{Co}\left(\mathrm{NO}_{3}\right)_{2} \cdot 6 \mathrm{H}_{2} \mathrm{O}\right)$; 氢氧化 钠 $(\mathrm{NaOH})$; 无水乙醇 $\left(\mathrm{CH}_{3} \mathrm{CH}_{2} \mathrm{OH}\right)$; 聚乙二醇 $\left(\mathrm{H}\left(\mathrm{OCH}_{2} \mathrm{CH}_{2}\right)_{n} \mathrm{OH}\right)$, 均为分析纯 $(\mathrm{AR})$ 。

实验仪器: DHG-9140A 型电热恒温鼓风干燥箱, 上海精宏实验设备有限公司; HHS 型恒温水浴锅, 上海博讯实业有限公司医疗设备厂; HJ-5 型多功能 搅拌器, 常州国华电器有限公司; SHZ-D(III)型循环 水式真空泵，南京艾科百业有限公司; KH-100 型水 热高压反应釜; HS.Z68 型电热蒸馏水器; JA2003 型 电子精密天平; CQF-50 型超声波清洗器; PHS-3E 型 数显 $\mathrm{pH}$ 计。

\subsection{GO 的制备}

采用改进 Hummers 法 ${ }^{[25-26]}$ 制备 GO: 将 $2 \mathrm{~g}$ 石 墨粉与 $46 \mathrm{~mL}$ 浓 $\mathrm{H}_{2} \mathrm{SO}_{4}$ 在冰水浴条件下混合, 充分 搅拌均匀。向其中缓慢加入 $6 \mathrm{~g} \mathrm{KMnO}_{4}$, 保持 $180 \mathrm{r} / \mathrm{min}$ 持续搅拌, $35^{\circ} \mathrm{C}$ 恒温水浴反应 $2 \mathrm{~h}$ 。缓慢滴 加 $92 \mathrm{~mL}$ 去离子水, 搅拌 $15 \mathrm{~min}$ 。逐滴加入 $280 \mathrm{~mL}$ 去离子水和 $10 \mathrm{~mL}$ 的 $\mathrm{H}_{2} \mathrm{O}_{2}$, 此时反应液呈亮黄色。 将反应液超声剥离 $30 \mathrm{~min}$ 后过滤。用 $500 \mathrm{~mL}$ 体积 分数为 $10 \%$ 的稀 $\mathrm{HCl}$ 去除金属离子, 静置 $2 \mathrm{~h}$, 移去 上清液, 用去离子水离心洗涤至中性, 超声剥离 $2 \mathrm{~h}$ 。超声后的分散液在离心机中 $3000 \mathrm{r} / \mathrm{min}$ 离心 $7 \mathrm{~min}$ 。将上层清液置于 $80{ }^{\circ} \mathrm{C}$ 的干燥箱中烘干、研 磨, 产物即为 $\mathrm{GO}$ 。

\section{$1.3 \mathrm{Co}_{0.5} \mathrm{Zn}_{0.5} \mathrm{Fe}_{2} \mathrm{O}_{4}$ 的制备}

按照化学计量比称取适量的 $\mathrm{Fe}\left(\mathrm{NO}_{3}\right)_{3} \cdot 9 \mathrm{H}_{2} \mathrm{O}$ 、 $\mathrm{Zn}\left(\mathrm{NO}_{3}\right)_{2} \cdot 6 \mathrm{H}_{2} \mathrm{O} 、 \mathrm{Co}\left(\mathrm{NO}_{3}\right)_{2} \cdot 6 \mathrm{H}_{2} \mathrm{O}$, 将以上原料溶解 于 $150 \mathrm{~mL}$ 蒸馏水中, 加入 $\mathrm{H}\left(\mathrm{OCH}_{2} \mathrm{CH}_{2}\right)_{\mathrm{n}} \mathrm{OH}$, 超声 处理 $30 \mathrm{~min}$ 分散均匀。得到的混合液置于四口烧瓶 中, 放入 $40{ }^{\circ} \mathrm{C}$ 恒温水浴箱中均匀搅拌, 以每 $2 \mathrm{~s}$ 一 滴的速率匀速向烧瓶中滴加 $2 \mathrm{~mol} / \mathrm{L}$ 的 $\mathrm{NaOH}$ 溶液, 同时调节溶液 $\mathrm{pH}$ 值至 10 , 在保持 $180 \mathrm{r} / \mathrm{min}$ 转速条 件下搅拌 $1 \mathrm{~h}$, 生成反应前驱体。常温下陈化 $8 \mathrm{~h}$ 静 
置分层, 移去部分上层清液, 将下层粘稠状反应前 驱体震荡摇匀移入带有聚四氟乙烯祄套的不锈钢水 热反应釜，将水热反应釜在 $180{ }^{\circ} \mathrm{C}$ 下进行 $8 \mathrm{~h}$ 晶化 反应，其中物料装填率为 $80 \%$ 。待反应结束自然冷 却，得到的物料先后用蒸馏水和无水乙醇各洗涤 4 次，洗净硝酸根离子，直至中性，真空抽滤，将滤 饼置于 $80{ }^{\circ} \mathrm{C}$ 电热恒温鼓风干燥箱中烘干，得到块 状物研磨至粉体，即得到纳米 $\mathrm{Co}_{0.5} \mathrm{Zn}_{0.5} \mathrm{Fe}_{2} \mathrm{O}_{4}$ 。

\section{$1.4 \mathrm{rGO} / \mathrm{Co}_{0.5} \mathrm{Zn}_{0.5} \mathrm{Fe}_{2} \mathrm{O}_{4}$ 复合材料的制备}

称量 $0.3 \mathrm{~g} \mathrm{GO}$ 置于 $1000 \mathrm{~mL}$ 烧杯中, 同时加入 $500 \mathrm{~mL}$ 蒸馏水, 超声震荡 $2 \mathrm{~h}$, 制备 $\mathrm{GO}$ 分散液。 $\mathrm{GO}: \mathrm{Co}_{0.5} \mathrm{Zn}_{0.5} \mathrm{Fe}_{2} \mathrm{O}_{4}$ 分别按质量比为 $1: 0.5 、 1: 1$ 、

$1: 2 、 1: 3$ 称取 $\mathrm{Co}_{0.5} \mathrm{Zn}_{0.5} \mathrm{Fe}_{2} \mathrm{O}_{4}$ 加入到 $\mathrm{GO}$ 分散液中 继续超声分散 $2 \mathrm{~h}$ 。将超声后的混合分散液移入带有 聚四氟乙烯祄套的不锈钢水热反应釜，再将水热反 应釜在 $180{ }^{\circ} \mathrm{C}$ 下进行 $12 \mathrm{~h}$ 晶化反应。待反应结束后 自然冷却, 得到的物料用蒸馏水洗涤, 真空抽滤.将 滤饼置于 $80{ }^{\circ} \mathrm{C}$ 电热恒温鼓风干燥箱中烘干, 得到块 状物研磨成粉体，制备粉体为 $\mathrm{rGO} / \mathrm{Co}_{0.5} \mathrm{Zn}_{0.5} \mathrm{Fe}_{2} \mathrm{O}_{4}$ 复合材料, 标记为 $\mathrm{rGO} / \mathrm{Co}_{0.5} \mathrm{Zn}_{0.5} \mathrm{Fe}_{2} \mathrm{O}_{4}-(1 \sim 4)$ 。

\section{5 物理及吸波性能表征}

采用 BRUKER 公司的 D8 ADVANCE 型 X 射线 衍射仪对样品进行物相组成分析, 测试条件: $\mathrm{Cu}$ 靶

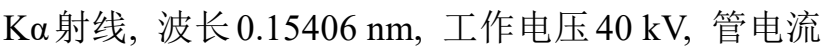
$30 \mathrm{~mA}$ ，扫描速度 $10^{\circ} / \mathrm{min}$ ，扫描范围 $5^{\circ} \sim 60^{\circ}$ 。用 Bragg 方程估算晶面间距 $(2 d \sin \theta=n \lambda, n=1,2, \cdots, d$ 为 晶面间距, $\theta$ 为入射 $X$ 射线与相应晶面的夹角, $\lambda$ 为 $X$ 射线的波长, $n$ 为衍射级数)。采用 BRUKER TENSOR II 傅立叶变换红外光谱仪对样品的官能团 进行测定, 波数范围 4000 350 $\mathrm{cm}^{-1}$, 扫描速度 $40 \mathrm{~S}^{-1}$, 分辨率为 $0.4 \mathrm{~cm}^{-1}$ 。用 JEOL 公司的 JEM-2010 型透 射电子显微镜(TEM)观察晶体的微观形貌及粒径大 小。Raman 测试采用美国 Thermo Scientific 公司 DXR 型拉曼光谱仪, 激发波长 $455 \mathrm{~nm}$, 功率 $6 \mathrm{~mW}$, 曝光时间 $20 \mathrm{~s}$, 波数范围 $250 \sim 3500 \mathrm{~cm}^{-1}$ 。

采用 Agilent 公司 HP8722ES 矢量网络分析仪 (VNA), 通过同轴线测试法对样品在 1 18 GHz 频率 范围的复介电常数和复磁导率进行测试。将样品粉 体与石蜡按 $1: 1$ 进行混合, 摚拌均匀, 将混合物压 制成外直径为 $7 \mathrm{~mm}$, 内径 $3 \mathrm{~mm}$, 厚度为 $2 \mathrm{~mm}$ 的圆 环状复合材料. 利用矢量网络分析仪测定样品的电 磁参数。根据吸波材料的电磁参数 $\left(\mu_{\mathrm{r}}, \varepsilon_{\mathrm{r}}\right)$ 测试结果, 并结合仪器参数和样品参数, 利用 MATLAB 软件 编程进行数值模拟计算, 绘制出相应的吸波反射率 与频率的关系曲线。

\section{2 结果与讨论}

\section{$2.1 \mathrm{rGO} / \mathrm{Co}_{0.5} \mathrm{Zn}_{0.5} \mathrm{Fe}_{2} \mathrm{O}_{4}$ 复合材料的微观结构}

图 1 是天然鳞片石墨、 $\mathrm{GO} 、 \mathrm{Co}_{0.5} \mathrm{Zn}_{0.5} \mathrm{Fe}_{2} \mathrm{O}_{4}$ 和 $\mathrm{rGO} / \mathrm{Co}_{0.5} \mathrm{Zn}_{0.5} \mathrm{Fe}_{2} \mathrm{O}_{4}$ 复合材料的 XRD 图谱。从图 1(a) 中可以看出, 天然鳞片石墨在 $2 \theta=26.51^{\circ}$ 出现了特 征峰, 归属于(002)晶面衍射, 根据 Bragg 方程计算 其层间距为 $0.34 \mathrm{~nm}^{[27-28]}$ 。从图 1(b)中可以看出, 天 然鳞片石墨经过氧化之后, 在 $2 \theta=9.74^{\circ}$ 处出现一个 尖锐的强衍射峰，而 $2 \theta=26.51^{\circ}$ 处的特征峰消失，说 明样品归属 $\mathrm{GO}(100)$ 晶面衍射, 其峰型规整, 峰宽 较窄, 表明样品具有良好的 GO 结构 ${ }^{[26]}$, 层间距为 $0.89 \mathrm{~nm}$ 。从图 1(c)中可以看出, $\mathrm{Co}_{0.5} \mathrm{Zn}_{0.5} \mathrm{Fe}_{2} \mathrm{O}_{4}$ 的衍 射峰分别对应 (111)、(220)、(311)、(400)、(422)、 (511) 晶面，与纯相的立方晶系尖晶石结构 (JCPDS 22-1086) 相符，表明实验分别合成了纯相的 $\mathrm{Co}_{0.5} \mathrm{Zn}_{0.5} \mathrm{Fe}_{2} \mathrm{O}_{4}$ 。产物的晶格常数 $a$ 可以根据下式进 行计算 ${ }^{[29]}$ :

$$
a=d\left(h^{2}+k^{2}+l^{2}\right)^{-1 / 2}
$$

式中: $d$ 为晶面间距; $(h k l)$ 为晶面指数。根据公式(1) 计算的 $\mathrm{Co}_{0.5} \mathrm{Zn}_{0.5} \mathrm{Fe}_{2} \mathrm{O}_{4}$ 和 $\mathrm{rGO} / \mathrm{Co}_{0.5} \mathrm{Zn}_{0.5} \mathrm{Fe}_{2} \mathrm{O}_{4}$ 复合 材料的晶格常数列于表 1 。从表 1 中可以看出两种 产物的(311)晶面对应的主衍射峰位置基本没有变 化, 晶格常数变化也不大，但是晶粒尺寸经过复合 反应过程后变大，这是因为在复合过程后 $\mathrm{Co}_{0.5} \mathrm{Zn}_{0.5} \mathrm{Fe}_{2} \mathrm{O}_{4}$ 的晶粒发生了团聚现象。从图 1(d) 中可以看出 $\mathrm{rGO} / \mathrm{Co}_{0.5} \mathrm{Zn}_{0.5} \mathrm{Fe}_{2} \mathrm{O}_{4}$ 复合材料中衍射峰 位置对应的晶面与图 1(c) $\mathrm{Co}_{0.5} \mathrm{Zn}_{0.5} \mathrm{Fe}_{2} \mathrm{O}_{4}$ 完全吻合, 而 $\mathrm{rGO} / \mathrm{Co}_{0.5} \mathrm{Zn}_{0.5} \mathrm{Fe}_{2} \mathrm{O}_{4}$ 复合材料在 $2 \theta=24.15^{\circ}$ 处衍射 峰则是 $\mathrm{GO}$ 在与 $\mathrm{Co}_{0.5} \mathrm{Zn}_{0.5} \mathrm{Fe}_{2} \mathrm{O}_{4}$ 复合过程中成功还 原为 $\mathrm{rGO}$ 而产生的特征衍射峰。这说明 $\mathrm{rGO}$ 与 $\mathrm{Co}_{0.5} \mathrm{Zn}_{0.5} \mathrm{Fe}_{2} \mathrm{O}_{4}$ 通过水热晶化复合后没有影响铁氧 体结构 ${ }^{[30]}$, 成功制备了 $\mathrm{rGO} / \mathrm{Co}_{0.5} \mathrm{Zn}_{0.5} \mathrm{Fe}_{2} \mathrm{O}_{4}$ 复合材料。

拉曼光谱是测定碳材料经化学处理后晶体结构 变化的一种快速准确的技术，也是描述 $\mathrm{sp}^{2}$ 碳材料无 序性的最灵敏和信息最丰富的技术之一 ${ }^{[31]}$ 。图 2 为天 然鳞片石墨、GO、 $\mathrm{Co}_{0.5} \mathrm{Zn}_{0.5} \mathrm{Fe}_{2} \mathrm{O}_{4}$ 和 $\mathrm{rGO} / \mathrm{Co}_{0.5} \mathrm{Zn}_{0.5} \mathrm{Fe}_{2} \mathrm{O}_{4}$ 复合材料的拉曼光谱。D 峰是由于六方石墨晶格的 破坏, 内部结构缺陷和边缘缺陷所致, 而 $\mathrm{G}$ 峰是由对 称 $\mathrm{sp}^{2} \mathrm{C}-\mathrm{C}$ 键在平面内伸缩运动而产生 ${ }^{[32]}$ 。从图 2(a) 中可以看出，天然鳞片石墨在 1354.17、1580.45、 2713.42 和 $3243.93 \mathrm{~cm}^{-1}$ 处有四个明显的峰, 分别 对应 D 峰、 G 峰、2D 峰和 $2 \mathrm{G}$ 峰; GO 在 1354.51、 $1604.08 \mathrm{~cm}^{-1}$ 处分别出现特征峰, 对应于 $D$ 峰和 $G$ 峰。通过对比, 可以明显发现天然鳞片石墨经过氧 

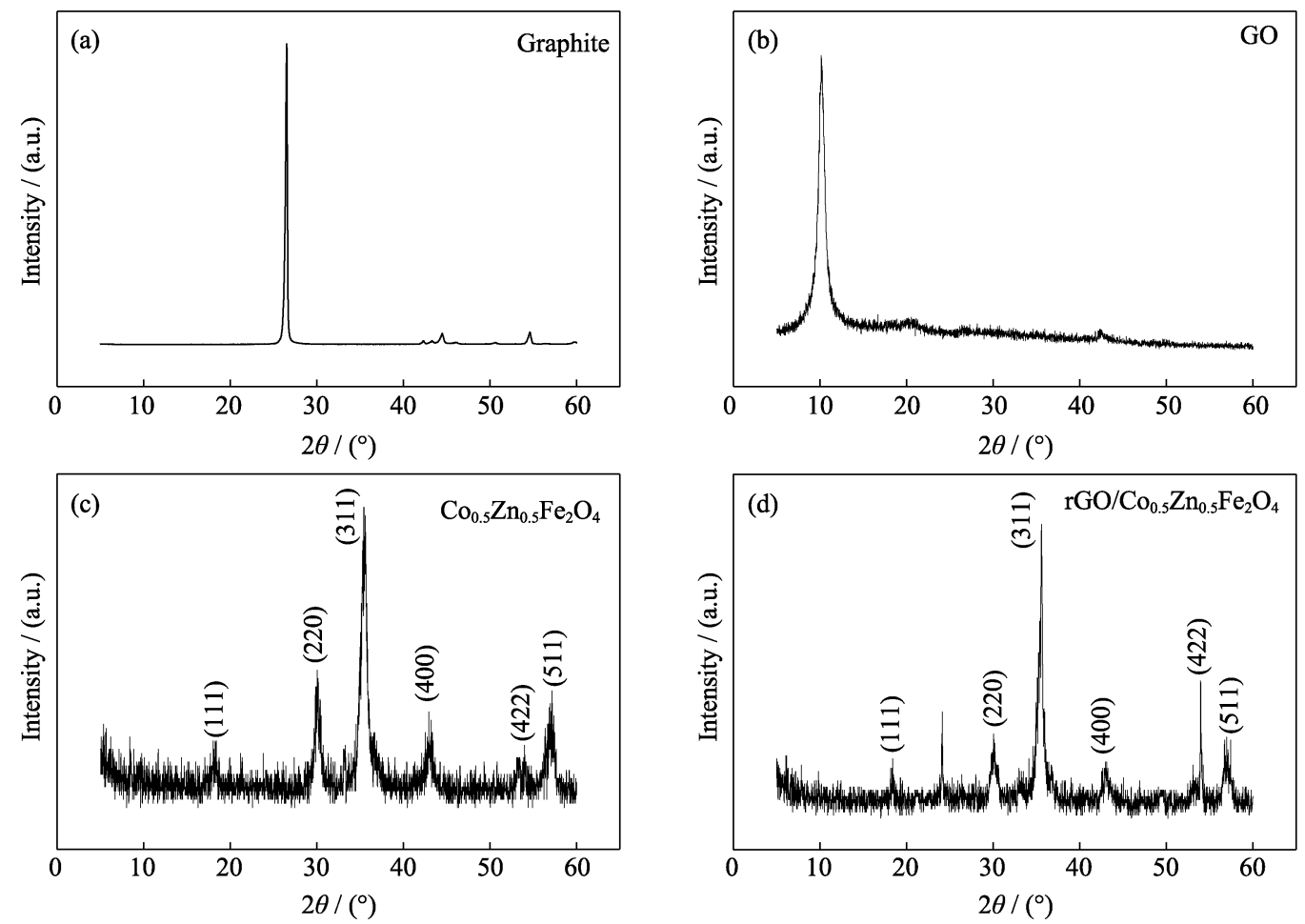

图 1 天然鳞片石墨(a)、 $\mathrm{GO}(\mathrm{b}) 、 \mathrm{Co}_{0.5} \mathrm{Zn}_{0.5} \mathrm{Fe}_{2} \mathrm{O}_{4}$ (c) 和 $\mathrm{rGO} / \mathrm{Co}_{0.5} \mathrm{Zn}_{0.5} \mathrm{Fe}_{2} \mathrm{O}_{4}$ 复合材料(d)的 XRD 图谱

Fig. 1 XRD patterns of natural flake graphite (a), $\mathrm{GO}(\mathrm{b}), \mathrm{Co}_{0.5} \mathrm{Zn}_{0.5} \mathrm{Fe}_{2} \mathrm{O}_{4}$ (c), and $\mathrm{rGO} / \mathrm{Co}_{0.5} \mathrm{Zn}_{0.5} \mathrm{Fe}_{2} \mathrm{O}_{4}$ composite (d)

表 1 铁氧体的组分和结构参数

Table 1 Composition and structure parameters of ferrite

\begin{tabular}{cccccc}
\hline \multirow{2}{*}{ Structural formula } & \multirow{2}{*}{$2 \theta /\left(^{\circ}\right)$} & $a / \mathrm{nm}$ & \multicolumn{3}{c}{ (311) Priority crystallization diffraction peak } \\
\cline { 4 - 6 } & & & FWHM/rad & Intensity/(a.u.) & Size/nm \\
\hline $\mathrm{Co}_{0.5} \mathrm{Zn}_{0.5} \mathrm{Fe}_{2} \mathrm{O}_{4}$ & 35.597 & 0.8391 & 0.618 & 86 & 13.8 \\
$\mathrm{rGO} / \mathrm{Co}_{0.5} \mathrm{Zn}_{0.5} \mathrm{Fe}_{2} \mathrm{O}_{4}$ & 35.595 & 0.8358 & 0.466 & 94 & 17.7 \\
\hline
\end{tabular}
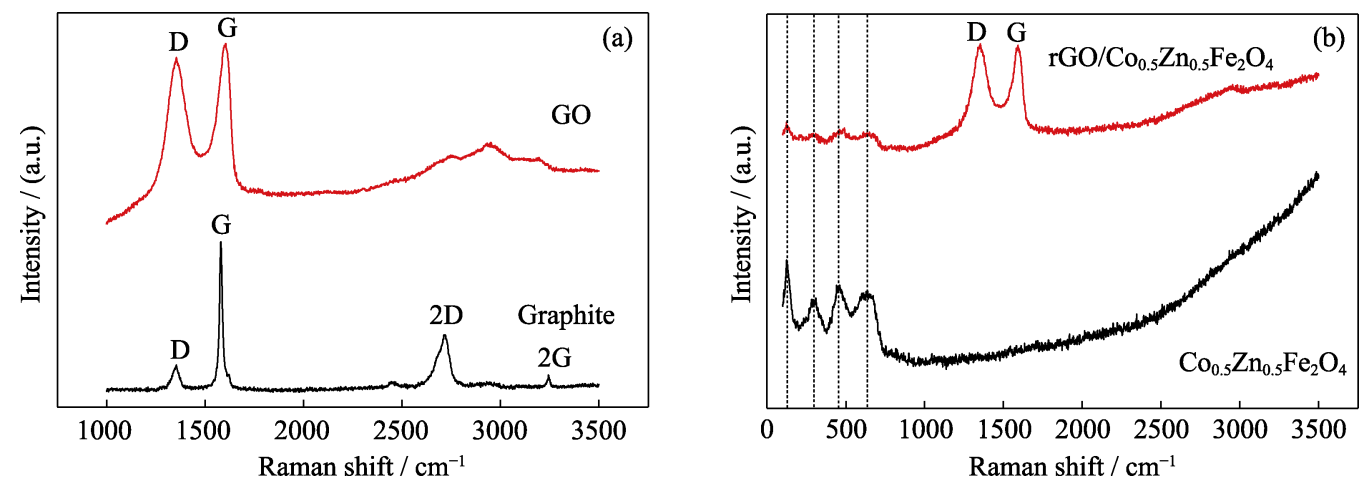

图 2 天然鳞片石墨、 $\mathrm{GO}(\mathrm{a})$ 和 $\mathrm{Co}_{0.5} \mathrm{Zn}_{0.5} \mathrm{Fe}_{2} \mathrm{O}_{4} 、 \mathrm{rGO} / \mathrm{Co}_{0.5} \mathrm{Zn}_{0.5} \mathrm{Fe}_{2} \mathrm{O}_{4}$ 复合材料(b)的拉曼光谱

Fig. 2 Raman spectra of natural flake graphite, $\mathrm{GO}$ (a) and $\mathrm{Co}_{0.5} \mathrm{Zn}_{0.5} \mathrm{Fe}_{2} \mathrm{O}_{4}, \mathrm{rGO} / \mathrm{Co}_{0.5} \mathrm{Zn}_{0.5} \mathrm{Fe}_{2} \mathrm{O}_{4}$ composite (b)

化生成 GO 的 D 峰迅速增大, G 峰发生宽化并由 $1580.45 \mathrm{~cm}^{-1}$ 移动至 $1604.08 \mathrm{~cm}^{-1}$ 处, 说明天然鳞片石 墨发生了极大程度的氧化 ${ }^{[33]}$ 。从 $\mathrm{rGO} / \mathrm{Co}_{0.5} \mathrm{Zn}_{0.5} \mathrm{Fe}_{2} \mathrm{O}_{4}$ 复合材料的拉曼光谱中可以看出, 在 1350.04 、 $1592.8 \mathrm{~cm}^{-1}$ 存在两个峰, 分别对应于 $D$ 峰和 $G$ 峰, 并在 123.84、308.59、460.56 和 $639.35 \mathrm{~cm}^{-1}$ 还存在 一些额外的峰, 这与 $\mathrm{Co}_{0.5} \mathrm{Zn}_{0.5} \mathrm{Fe}_{2} \mathrm{O}_{4}$ 的拉曼光谱分
析结果吻合得很好, 说明 $\mathrm{Co}_{0.5} \mathrm{Zn}_{0.5} \mathrm{Fe}_{2} \mathrm{O}_{4}$ 已经负载 到 $\mathrm{rGO}$ 上。

$\mathrm{D}$ 峰和 $\mathrm{G}$ 峰的强度比 $\left(I_{\mathrm{D}} / I_{\mathrm{G}}\right)$ 是衡量 $\mathrm{sp}^{2}$ 结构域无 序程度和平均大小的重要指标 ${ }^{[34]}$ 。表 2 列出了不同 样品拉曼光谱的 $D$ 峰与 $G$ 峰强度的比值。从表 2 中可以看到, 天然鳞片石墨经过氧化后, $I_{\mathrm{D}} / I_{\mathrm{G}}$ 从 0.21 增加到 0.95 , 表明石墨被氧化良好, 这与 XRD 
表 2 天然鳞片石墨、 $\mathrm{GO}$ 和 $\mathrm{rGO} / \mathrm{Co}_{0.5} \mathrm{Zn}_{0.5} \mathrm{Fe}_{2} \mathrm{O}_{4}$ 复合材料的 $I_{\mathrm{D}}$ 值与 $I_{\mathrm{G}}$ 值的比率

Table $2 I_{\mathrm{D}} / I_{\mathrm{G}}$ ratios of natural flake graphite, $\mathrm{GO}$ and $\mathrm{rGO} / \mathrm{Co}_{0.5} \mathrm{Zn}_{0.5} \mathrm{Fe}_{2} \mathrm{O}_{4}$ composite

\begin{tabular}{cccc}
\hline Samples & Graphite & $\mathrm{GO}$ & $\mathrm{rGO} / \mathrm{Co}_{0.5} \mathrm{Zn}_{0.5} \mathrm{Fe}_{2} \mathrm{O}_{4}$ \\
\hline$I_{\mathrm{D}} / I_{\mathrm{G}}$ & 0.21 & 0.95 & 1.02 \\
\hline
\end{tabular}

Notes: $I_{\mathrm{D}}$-Intensity of $\mathrm{D}$ peaks; $I_{\mathrm{G}}$-Intensity of $\mathrm{G}$ peaks; $I_{\mathrm{D}} / I_{\mathrm{G}}$ - Indicator to evaluate the disorder degree and average size of the $\mathrm{sp}^{2}$ domains

分析结果一致。 $\mathrm{rGO} / \mathrm{Co}_{0.5} \mathrm{Zn}_{0.5} \mathrm{Fe}_{2} \mathrm{O}_{4}$ 的 $I_{\mathrm{D}} / I_{\mathrm{G}}$ 值为 1.02 , 比 GO 有所增大, 这是由于将 $\mathrm{Co}_{0.5} \mathrm{Zn}_{0.5} \mathrm{Fe}_{2} \mathrm{O}_{4}$ 复合 $\mathrm{rGO}$ 之后在 $\mathrm{sp}^{2}$ 碳网络中存在局域化的 $\mathrm{sp}^{3}$ 缺陷, 进 一步增加了石墨晶粒的数量, 这可能是由于加入 $\mathrm{Co}_{0.5} \mathrm{Zn}_{0.5} \mathrm{Fe}_{2} \mathrm{O}_{4}$ 后 $\mathrm{GO}$ 的有序石墨晶体结构的显著 下降所致 ${ }^{[35]}$ 。同时也说明在水热反应过程中 $\mathrm{GO}$ 被 还原且 $\mathrm{sp}^{2}$ 结构域平均尺寸减小 ${ }^{[36]}$ 。以上均说明天然 鳞片石墨首先被氧化为 $\mathrm{GO}$, 之后与 $\mathrm{Co}_{0.5} \mathrm{Zn}_{0.5} \mathrm{Fe}_{2} \mathrm{O}_{4}$ 进行复合, 成功制备了 $\mathrm{rGO} / \mathrm{Co}_{0.5} \mathrm{Zn}_{0.5} \mathrm{Fe}_{2} \mathrm{O}_{4}$ 复合材料。

\section{$2.2 \mathrm{rGO} / \mathrm{Co}_{0.5} \mathrm{Zn}_{0.5} \mathrm{Fe}_{2} \mathrm{O}_{4}$ 复合材料的官能团}

图 3 为天然鳞片石墨、 $\mathrm{GO}(\mathrm{a}) 、 \mathrm{Co}_{0.5} \mathrm{Zn}_{0.5} \mathrm{Fe}_{2} \mathrm{O}_{4}(\mathrm{~b})$ 和 $\mathrm{rGO} / \mathrm{Co}_{0.5} \mathrm{Zn}_{0.5} \mathrm{Fe}_{2} \mathrm{O}_{4}$ 复合材料(c)的红外光谱图。 在天然鳞片石墨的红外光谱图中几乎没有观察到石 墨峰值, 经过氧化反应后, 出现了一些强的吸收峰。 GO 的红外光谱中, $3404.60 \mathrm{~cm}^{-1}$ 处的峰是由于 $\mathrm{H}_{2} \mathrm{O} / \mathrm{O}-\mathrm{H}$ 振动变形所产生的; $1626.54 \mathrm{~cm}^{-1}$ 处的峰 是由于羰基 $(\mathrm{C}=\mathrm{O})$ 的伸缩振动所产生; $1400.65 \mathrm{~cm}^{-1}$ 处的峰是由于 $\mathrm{C}=\mathrm{C}$ 振动所产生的吸收峰; $1225.62 \mathrm{~cm}^{-1}$ 处的峰是由于 $\mathrm{C}-\mathrm{OH}$ 的价键振动所产 生; $1062.83 \mathrm{~cm}^{-1}$ 处的峰是由于环氧基振动所产生的 峰位 ${ }^{[37]}$, 这说明天然鳞片石墨经过氧化生成了 $\mathrm{GO}$ 。 然而, 由图 3(c) 可以看出经过水热晶化后所有与 GO 含氧官能团有关的峰几乎消失, 表明这些含氧 官能团在 $\mathrm{rGO}$ 与 $\mathrm{Co}_{0.5} \mathrm{Zn}_{0.5} \mathrm{Fe}_{2} \mathrm{O}_{4}$ 复合的过程中被移 除 ${ }^{[38]}$ 。在 2000 和 $2250 \mathrm{~cm}^{-1}$ 处产生的新吸收带是由 于石墨烯片的骨架振动所产生的 ${ }^{[39-41]}$, 而其余的峰 与 $\mathrm{Co}_{0.5} \mathrm{Zn}_{0.5} \mathrm{Fe}_{2} \mathrm{O}_{4}$ 的红外光谱所对应, 证实了 $\mathrm{rGO} /$ $\mathrm{Co}_{0.5} \mathrm{Zn}_{0.5} \mathrm{Fe}_{2} \mathrm{O}_{4}$ 复合材料中 $\mathrm{Co}_{0.5} \mathrm{Zn}_{0.5} \mathrm{Fe}_{2} \mathrm{O}_{4}$ 的存在。 以上均说明实验成功制备了 $\mathrm{rGO} / \mathrm{Co}_{0.5} \mathrm{Zn}_{0.5} \mathrm{Fe}_{2} \mathrm{O}_{4}$ 复 合材料。这与 XRD 和拉曼光谱的表征结果一致。

\section{$2.3 \mathrm{rGO} / \mathrm{Co}_{0.5} \mathrm{Zn}_{0.5} \mathrm{Fe}_{2} \mathrm{O}_{4}$ 复合材料的微观形貌}

图 4 为制备的不同比例 $\mathrm{rGO} / \mathrm{Co}_{0.5} \mathrm{Zn}_{0.5} \mathrm{Fe}_{2} \mathrm{O}_{4}$ 复 合材料的 TEM 照片, 从图中可以看出经过晶化复 合后的 $\mathrm{rGO}$ 呈现为透明薄膜。图 4(a)为 $\mathrm{rGO} /$ $\mathrm{Co}_{0.5} \mathrm{Zn}_{0.5} \mathrm{Fe}_{2} \mathrm{O}_{4}-1$ 的透射电子显微镜照片, 从图中 可以看出有几层 $\mathrm{rGO}$ 堆叠在一起, 并且在旁边存在 散落的 $\mathrm{Co}_{0.5} \mathrm{Zn}_{0.5} \mathrm{Fe}_{2} \mathrm{O}_{4}$ 颗粒，这是由于 $\mathrm{Co}_{0.5} \mathrm{Zn}_{0.5} \mathrm{Fe}_{2} \mathrm{O}_{4}$
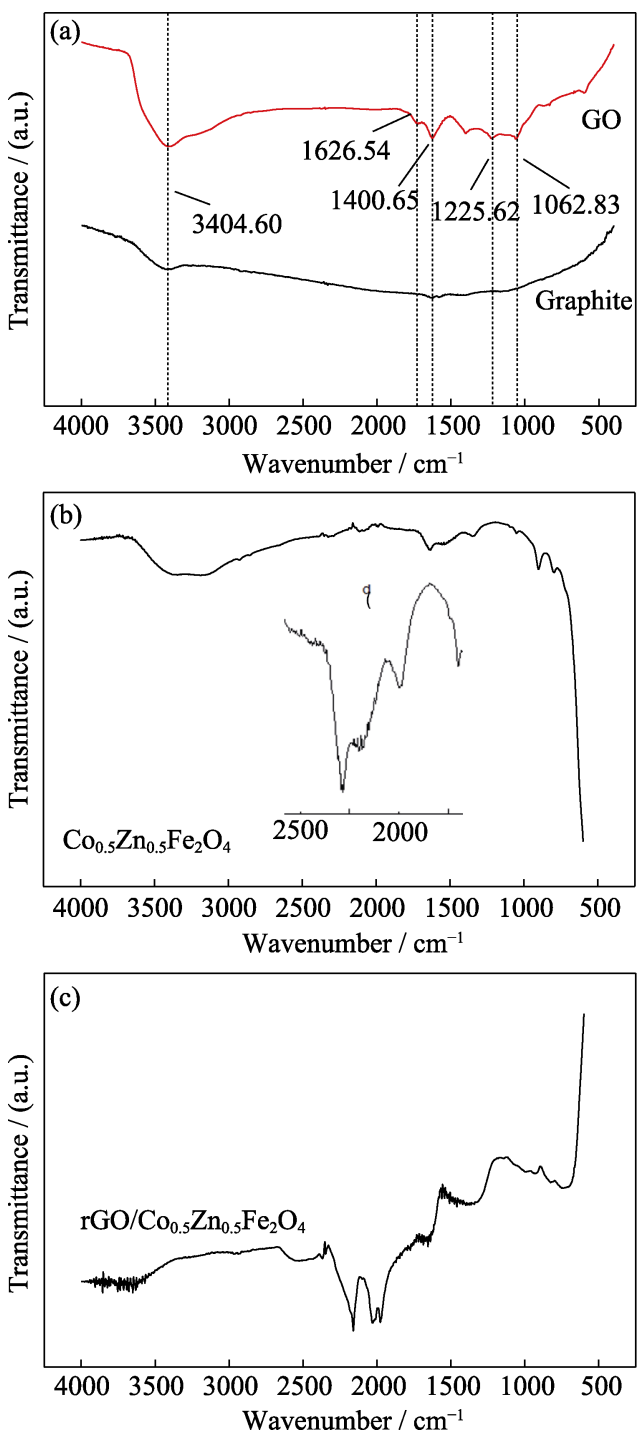

图 3 天然鳞片石墨、 $\mathrm{GO}$ (a)、 $\mathrm{Co}_{0.5} \mathrm{Zn}_{0.5} \mathrm{Fe}_{2} \mathrm{O}_{4}$ (b) 和 $\mathrm{rGO} / \mathrm{Co}_{0.5} \mathrm{Zn}_{0.5} \mathrm{Fe}_{2} \mathrm{O}_{4}$ (c) 的 FT-IR 谱图

Fig. 3 FT-IR spectra of natural flake graphite, GO (a), $\mathrm{Co}_{0.5} \mathrm{Zn}_{0.5} \mathrm{Fe}_{2} \mathrm{O}_{4}$ (b) and $\mathrm{rGO} / \mathrm{Co}_{0.5} \mathrm{Zn}_{0.5} \mathrm{Fe}_{2} \mathrm{O}_{4}$ (c)

占的比例较低, $\mathrm{rGO}$ 在高温高压的环境下发生聚集。 图 4(b) (d) 分别是 $\mathrm{rGO} / \mathrm{Co}_{0.5} \mathrm{Zn}_{0.5} \mathrm{Fe}_{2} \mathrm{O}_{4}-2 、 \mathrm{rGO} /$ $\mathrm{Co}_{0.5} \mathrm{Zn}_{0.5} \mathrm{Fe}_{2} \mathrm{O}_{4}-3 、 \mathrm{rGO} / \mathrm{Co}_{0.5} \mathrm{Zn}_{0.5} \mathrm{Fe}_{2} \mathrm{O}_{4}-4$ 的透射电 子显微镜照片, 可以看出, 随着 $\mathrm{Co}_{0.5} \mathrm{Zn}_{0.5} \mathrm{Fe}_{2} \mathrm{O}_{4}$ 所 占比例的增大, $\mathrm{rGO}$ 表面分布的 $\mathrm{Co}_{0.5} \mathrm{Zn}_{0.5} \mathrm{Fe}_{2} \mathrm{O}_{4}$ 越来 越密集，这些 $\mathrm{Co}_{0.5} \mathrm{Zn}_{0.5} \mathrm{Fe}_{2} \mathrm{O}_{4}$ 颗粒牢固地附着在 $\mathrm{rGO}$ 薄片上，说明 $\mathrm{rGO}$ 和 $\mathrm{Co}_{0.5} \mathrm{Zn}_{0.5} \mathrm{Fe}_{2} \mathrm{O}_{4}$ 之间具有优 异的附着力。研究显示, GO 还原后会产生大量的化 学和物理缺陷, 使 $\mathrm{Co}_{0.5} \mathrm{Zn}_{0.5} \mathrm{Fe}_{2} \mathrm{O}_{4}$ 颗粒可以附着在 这些缺陷上 ${ }^{[35]}$ 。但是随着 $\mathrm{Co}_{0.5} \mathrm{Zn}_{0.5} \mathrm{Fe}_{2} \mathrm{O}_{4}$ 所占比例的 提高, $\mathrm{Co}_{0.5} \mathrm{Zn}_{0.5} \mathrm{Fe}_{2} \mathrm{O}_{4}$ 在 $\mathrm{rGO}$ 上的分散性逐渐减弱。

\section{$2.4 \mathrm{rGO} / \mathrm{Co}_{0.5} \mathrm{Zn}_{0.5} \mathrm{Fe}_{2} \mathrm{O}_{4}$ 复合材料介电常数} 和磁导率

图 5 为 $\mathrm{GO} 、 \mathrm{Co}_{0.5} \mathrm{Zn}_{0.5} \mathrm{Fe}_{2} \mathrm{O}_{4}$ 、不同比例 $\mathrm{rGO}$ / $\mathrm{Co}_{0.5} \mathrm{Zn}_{0.5} \mathrm{Fe}_{2} \mathrm{O}_{4}$ 复合材料在 $1 \sim 18 \mathrm{GHz}$ 范围内测量的 

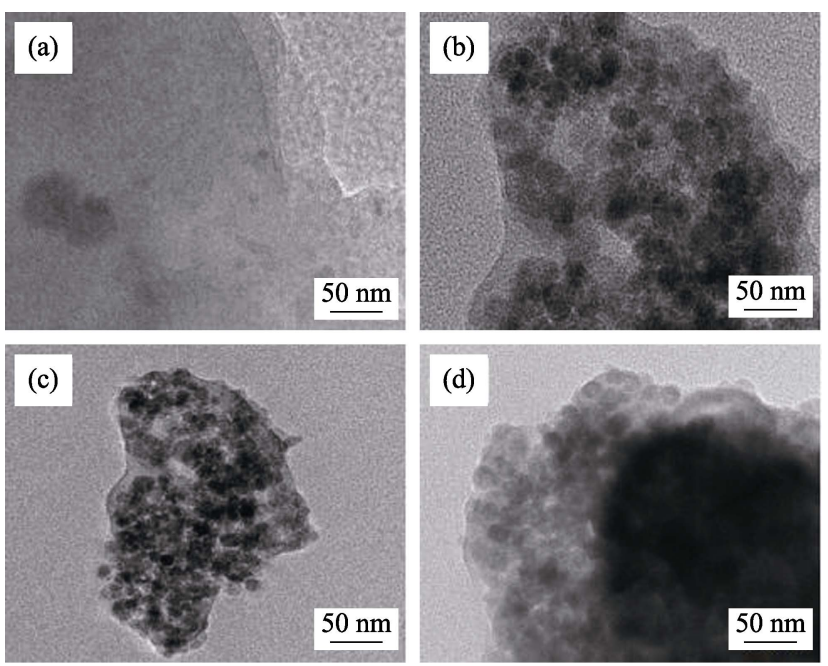

图 $4 \mathrm{rGO} / \mathrm{Co}_{0.5} \mathrm{Zn}_{0.5} \mathrm{Fe}_{2} \mathrm{O}_{4}-1(\mathrm{a}) 、 \mathrm{rGO} / \mathrm{Co}_{0.5} \mathrm{Zn}_{0.5} \mathrm{Fe}_{2} \mathrm{O}_{4}-2(\mathrm{~b})$ 、 $\mathrm{rGO} / \mathrm{Co}_{0.5} \mathrm{Zn}_{0.5} \mathrm{Fe}_{2} \mathrm{O}_{4}-3(\mathrm{c})$ 和 $\mathrm{rGO} / \mathrm{Co}_{0.5} \mathrm{Zn}_{0.5} \mathrm{Fe}_{2} \mathrm{O}_{4}-4(\mathrm{~d})$ 的 TEM 照片

Fig. 4 TEM images of $\mathrm{rGO} / \mathrm{Co}_{0.5} \mathrm{Zn}_{0.5} \mathrm{Fe}_{2} \mathrm{O}_{4}-1(\mathrm{a}), \mathrm{rGO} /$ $\mathrm{Co}_{0.5} \mathrm{Zn}_{0.5} \mathrm{Fe}_{2} \mathrm{O}_{4}-2$ (b), $\mathrm{rGO} / \mathrm{Co}_{0.5} \mathrm{Zn}_{0.5} \mathrm{Fe}_{2} \mathrm{O}_{4}-3$ (c), and $\mathrm{rGO} /$ $\mathrm{Co}_{0.5} \mathrm{Zn}_{0.5} \mathrm{Fe}_{2} \mathrm{O}_{4}-4$ (d)

复介电常数实部(a)、复介电常数虚部(b)、磁导率实 部(c)、磁导率虚部(d)、介电损耗(e)和磁滞损耗(f)。 从图 5(a)中可以看出, $\mathrm{GO}$ 的复介电常数实部的起始 值为 5.54, 随着频率的增加, $\varepsilon^{\prime}$ 的值下降为 4.11 。而 $\mathrm{Co}_{0.5} \mathrm{Zn}_{0.5} \mathrm{Fe}_{2} \mathrm{O}_{4}$ 的起始值为 4.81 , 当频率增加变为 5.12 。随着 $\mathrm{Co}_{0.5} \mathrm{Zn}_{0.5} \mathrm{Fe}_{2} \mathrm{O}_{4}$ 的引入，按照复合比例逐 渐增大的顺序, 复介电常数实部的起始值均呈现增
加状态，依次为 $14.85 、 13.43 、 14.58 、 18.66$, 并且 随着频率升高均产生下降，依次为 $10.31 、 10.85$ 、

$12.18 、 12.64$ 。再由图 5(b) 可以看到, $\mathrm{GO}$ 的 $\varepsilon^{\prime \prime}$ 从 1.32 下降为 $0.68 、 \mathrm{Co}_{0.5} \mathrm{Zn}_{0.5} \mathrm{Fe}_{2} \mathrm{O}_{4}$ 的 $\varepsilon^{\prime \prime}$ 从 0.05 上升为 0.31。随着 $\mathrm{Co}_{0.5} \mathrm{Zn}_{0.5} \mathrm{Fe}_{2} \mathrm{O}_{4}$ 的比例逐渐增加, 复合材 料的 $\varepsilon^{\prime \prime}$ 的起始值依次为 $3.12 、 2.2 、 2.24 、 5.92$, 随 着频率的不断升高，终止值依次变为 3.91、2.94、 5.2、4.36。通过对比可以看出, 单一 $\mathrm{GO}$ 或者 $\mathrm{Co}_{0.5} \mathrm{Zn}_{0.5} \mathrm{Fe}_{2} \mathrm{O}_{4}$ 的复介电常数都很低, 将两者按照 不同的比例复合后, 复介电常数均有所升高, 表明 复合材料具有较高的导电性。

根据自由电子理论 ${ }^{[22]}$ :

$$
\varepsilon^{\prime \prime}=\frac{1}{2} \varepsilon_{0} \pi \rho f
$$

其中 $\varepsilon_{0}$ 为真空的介电常数, $\rho$ 为电阻率, $f$ 是电磁波的 频率。在复合反应过程中 $\mathrm{GO}$ 还原为 $\mathrm{rGO}$, 而 $\mathrm{rGO}$ 的电导率较高, 形成了较大的导电网络, 使复合材 料的电阻率 $\rho$ 降低。说明 $\mathrm{rGO}$ 与 $\mathrm{Co}_{0.5} \mathrm{Zn}_{0.5} \mathrm{Fe}_{2} \mathrm{O}_{4}$ 复 合可以改善材料的复介电常数, 其中 $\mathrm{rGO} /$ $\mathrm{Co}_{0.5} \mathrm{Zn}_{0.5} \mathrm{Fe}_{2} \mathrm{O}_{4}-3$ 表现出较高的复介电常数。

从图 5(c)中可以看出随着频率逐渐增大, $\mathrm{GO}$ 的 磁导率实部 $\mu^{\prime}$ 从 1.05 变为 1 , 几乎没有什么变化, 而 $\mathrm{Co}_{0.5} \mathrm{Zn}_{0.5} \mathrm{Fe}_{2} \mathrm{O}_{4}$ 的 $\mu^{\prime}$ 从 1.3 下降为 1.09 。此时将 $\mathrm{Co}_{0.5} \mathrm{Zn}_{0.5} \mathrm{Fe}_{2} \mathrm{O}_{4}$ 按比例增大顺序引入, $\mu^{\prime}$ 依次从 $0.97 、 1.06 、 1.06 、 1.12$ 变为 $0.92 、 0.95 、 0.94 、 1$, 均
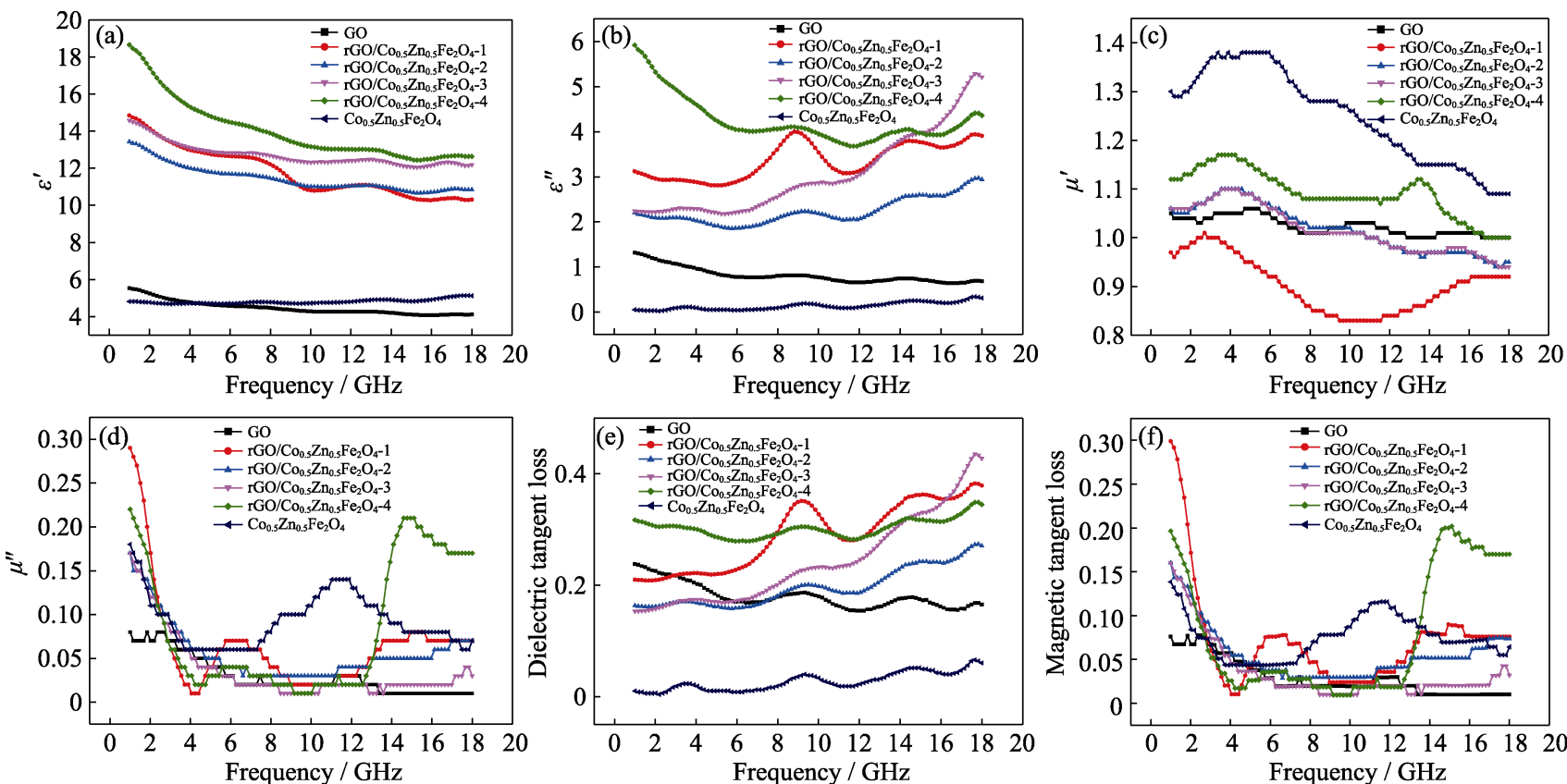

图 $5 \mathrm{GO} 、 \mathrm{Co}_{0.5} \mathrm{Zn}_{0.5} \mathrm{Fe}_{2} \mathrm{O}_{4}$ 和不同比例的 $\mathrm{rGO} / \mathrm{Co}_{0.5} \mathrm{Zn}_{0.5} \mathrm{Fe}_{2} \mathrm{O}_{4}$ 复合材料的复介电常数实部(a)、 复介电常数虚部(b)、磁导率实部 $(c)$ 、磁导率虚部 $(d)$ 、介电损耗 $(e)$ 和磁滞损耗(f)

Fig. 5 Real part of complex permittivity (a), imaginary part of complex permittivity (b), real part of complex permeability (c), imaginary part of complex permeability (d), dielectric loss tangent (e) and magnetic loss tangent (f) of $\mathrm{GO} 、 \mathrm{Co}_{0.5} \mathrm{Zn}_{0.5} \mathrm{Fe}_{2} \mathrm{O}_{4}$ and $\mathrm{rGO} / \mathrm{Co}_{0.5} \mathrm{Zn}_{0.5} \mathrm{Fe}_{2} \mathrm{O}_{4}$ composite with different ratios 

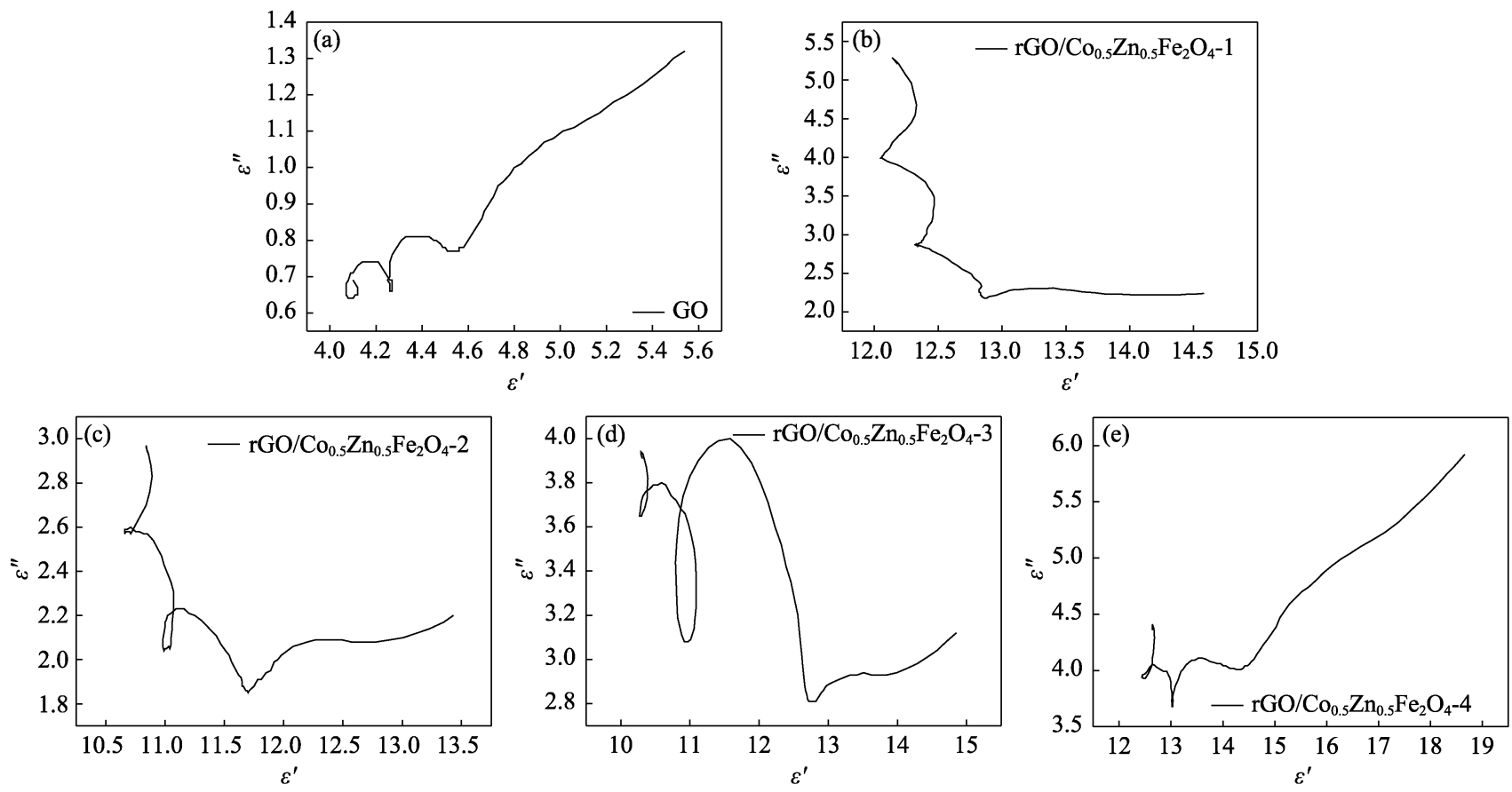

图 $6 \mathrm{GO}$ 和不同复合比例 $\mathrm{rGO} / \mathrm{Co}_{0.5} \mathrm{Zn}_{0.5} \mathrm{Fe}_{2} \mathrm{O}_{4}$ 复合材料的复介电常数 $\left(\varepsilon^{\prime}\right)$-复介电常数虚部 $\left(\varepsilon^{\prime \prime}\right)$ 曲线

Fig. 6 Real part of complex permittivity $\left(\varepsilon^{\prime}\right)$-imaginary part of complex permittivity $\left(\varepsilon^{\prime \prime}\right)$ curves of GO and $\mathrm{rGO} / \mathrm{Co}_{0.5} \mathrm{Zn}_{0.5} \mathrm{Fe}_{2} \mathrm{O}_{4}$ composites with different ratios

呈现出不同程度的下降, 这与 $\mathrm{Co}_{0.5} \mathrm{Zn}_{0.5} \mathrm{Fe}_{2} \mathrm{O}_{4}$ 的变 化趋势一致。再看磁导率虚部 $\mu^{\prime \prime}$ 曲线, 图 5(d)中 GO 的 $\mu^{\prime \prime}$ 从 0.08 下降为 0.01 , 钴锌铁氧体的 $\mu^{\prime \prime}$ 从 0.18 下降为 0.07 。随着复合材料中 $\mathrm{Co}_{0.5} \mathrm{Zn}_{0.5} \mathrm{Fe}_{2} \mathrm{O}_{4}$ 的比 例逐渐增大, 曲线整体趋势呈现出先下降后上升, 可以看出 $\mathrm{rGO} / \mathrm{Co}_{0.5} \mathrm{Zn}_{0.5} \mathrm{Fe}_{2} \mathrm{O}_{4}-3$ 的 $\mu^{\prime \prime}$ 值最小, 而 $\mathrm{rGO} / \mathrm{Co}_{0.5} \mathrm{Zn}_{0.5} \mathrm{Fe}_{2} \mathrm{O}_{4}-4$ 却表现出较高的磁导率。微波 吸收性能的优异与否有两个衡量标准, 即介电损耗 和磁滞损耗。图 5(e)和(f)为 $\mathrm{GO} 、 \mathrm{Co}_{0.5} \mathrm{Zn}_{0.5} \mathrm{Fe}_{2} \mathrm{O}_{4}$ 、 不同比例 $\mathrm{rGO} / \mathrm{Co}_{0.5} \mathrm{Zn}_{0.5} \mathrm{Fe}_{2} \mathrm{O}_{4}$ 复合材料的介电损耗 $\left(\tan \delta_{\mathrm{E}}=\varepsilon^{\prime \prime} / \varepsilon^{\prime}\right)$ 和磁滞损耗 $\left(\tan \delta_{\mathrm{M}}=\mu^{\prime \prime} / \mu^{\prime}\right)$ 的曲线, 频率 在 1 18 GHz 之间, $\mathrm{GO}$ 的 $\tan \delta_{\mathrm{E}}$ 从 0.23 下降到 0.16 , $\tan \delta_{\mathrm{M}}$ 从 0.07 下降为 0.01 ; 而 $\mathrm{Co}_{0.5} \mathrm{Zn}_{0.5} \mathrm{Fe}_{2} \mathrm{O}_{4}$ 的 $\tan \delta_{\mathrm{E}}$ 从 0.01 上升到 $0.06 、 \tan \delta_{\mathrm{M}}$ 从 0.13 下降为 0.06 。观 察复合材料的介电损耗和磁滞损耗曲线, 发现 $\mathrm{rGO} / \mathrm{Co}_{0.5} \mathrm{Zn}_{0.5} \mathrm{Fe}_{2} \mathrm{O}_{4}-3$ 的介电损耗随着频率的增加 而逐渐增大, 并在 $17 \mathrm{GHz}$ 左右达到最大, 超过其他 样品。从图 5(e)中可以明显看到 $\mathrm{rGO} / \mathrm{Co}_{0.5} \mathrm{Zn}_{0.5} \mathrm{Fe}_{2} \mathrm{O}_{4}-1$ 在 $9 \mathrm{GHz}$ 左右产生一个峰, 这是两种粒子的共 振行为产生的 ${ }^{[35]}$ 。从图 5(f)中可以看到, 引入 $\mathrm{Co}_{0.5} \mathrm{Zn}_{0.5} \mathrm{Fe}_{2} \mathrm{O}_{4}$ 后, 样品的磁损耗相比于 $\mathrm{GO}$ 确实有 一定的提升, $\mathrm{rGO} / \mathrm{Co}_{0.5} \mathrm{Zn}_{0.5} \mathrm{Fe}_{2} \mathrm{O}_{4}-1$ 在 $6 \mathrm{GHz}$ 左右处 产生一个峰, 这是因为复合材料中 $\mathrm{Co}_{0.5} \mathrm{Zn}_{0.5} \mathrm{Fe}_{2} \mathrm{O}_{4}$ 含量较少, 而 rGO 本身含有巡游电子, 会发生巡游 电子的自旋振荡以及局域电子和巡游电子产生反铁
磁耦合作用, 从而产生峰位 ${ }^{[43]}$ 。 $\mathrm{Co}_{0.5} \mathrm{Zn}_{0.5} \mathrm{Fe}_{2} \mathrm{O}_{4}$ 在 $11 \mathrm{GHz}$ 左右处产生的峰是 $\mathrm{Co}_{0.5} \mathrm{Zn}_{0.5} \mathrm{Fe}_{2} \mathrm{O}_{4}$ 的小尺寸 效应和表面效应共同作用的结 果 ${ }^{[44]}$ 。 $\mathrm{rGO} / \mathrm{Co}_{0.5} \mathrm{Zn}_{0.5} \mathrm{Fe}_{2} \mathrm{O}_{4}-4$ 在 $16 \mathrm{GHz}$ 处产生的峰是由于 复合材料中 $\mathrm{Co}_{0.5} \mathrm{Zn}_{0.5} \mathrm{Fe}_{2} \mathrm{O}_{4}$ 含量较高, 在装载过程中 $\mathrm{Co}_{0.5} \mathrm{Zn}_{0.5} \mathrm{Fe}_{2} \mathrm{O}_{4}$ 难免发生团聚导致粒子的半径增大, 而自旋波激发的共振频率恰恰依赖于粒子的半径, 使样品在 $16 \mathrm{GHz}$ 处产生自然共振, 在曲线上表现 为强峰 ${ }^{[35]}$ 。从图 5(e) (f) 明显看出, 样品的 $\tan \delta_{\mathrm{E}}$ 要 大于 $\tan \delta_{\mathrm{M}}$, 说明反射损耗主要是由于介电损耗产生。

\section{$2.5 \mathrm{rGO} / \mathrm{Co}_{0.5} \mathrm{Zn}_{0.5} \mathrm{Fe}_{2} \mathrm{O}_{4}$ 复合材料的德拜弛} 豫模型

用 Cole-Cole 半圆描述的弛豫过程对微波吸收 材料的介电常数行为有很大的影响, 由德拜偶极弛 豫, 相对复介电常数可用下列方程表示 ${ }^{[45]}$ :

$$
\varepsilon_{\mathrm{r}}=\varepsilon_{\infty}+\frac{\varepsilon_{\mathrm{s}}-\varepsilon_{\infty}}{1+\mathrm{j} 2 \pi f \tau}=\varepsilon^{\prime}(f)+\mathrm{i} \varepsilon^{\prime \prime}(f)
$$

其中, $f$ 为频率, $\tau$ 为弛豫时间, $\varepsilon_{\mathrm{s}}$ 和 $\varepsilon_{\infty}$ 分别为固定介 电常数和光学介电常数, 由(3)可以推断:

$$
\begin{gathered}
\varepsilon^{\prime}(f)=\varepsilon_{\infty}+\frac{\varepsilon_{\mathrm{s}}-\varepsilon_{\infty}}{1+(2 \pi f)^{2} \tau^{2}} \\
\varepsilon^{\prime \prime}(f)=\frac{2 \pi f \tau\left(\varepsilon_{\mathrm{s}}-\varepsilon_{\infty}\right)}{1+(2 \pi f)^{2} \tau^{2}}
\end{gathered}
$$

根据公式(4)和(5), 可以推出 $\varepsilon^{\prime}$ 和 $\varepsilon^{\prime \prime}$ 之间的关 系为: 


$$
\left(\varepsilon^{\prime}-\varepsilon_{\infty}\right)^{2}+\left(\varepsilon^{\prime \prime}\right)^{2}=\left(\varepsilon_{\mathrm{s}}-\varepsilon_{\infty}\right)^{2}
$$

以此数学方程为基础, 得出 $\varepsilon^{\prime}-\varepsilon^{\prime \prime}$ 曲线表示单个半圆, 通常定义为 Cole-Cole 半圆, 每个半圆对应于一个 德拜弛豫过程。图 6 为 $\mathrm{GO}$ 和不同复合比例 $\mathrm{rGO} /$ $\mathrm{Co}_{0.5} \mathrm{Zn}_{0.5} \mathrm{Fe}_{2} \mathrm{O}_{4}$ 复合材料的介电 Cole-Cole 半圆, 从 图中可以看出, 当 $\mathrm{GO}$ 与 $\mathrm{Co}_{0.5} \mathrm{Zn}_{0.5} \mathrm{Fe}_{2} \mathrm{O}_{4}$ 复合发生还原 反应而生成 $\mathrm{rGO}$ 后弛豫效果明显加强, 说明 $\mathrm{rGO}$ 的 存在提高了德拜偶极弛豫过程的强度; 并且复合材料 中有两个明显重叠的半圆, 说明 $\mathrm{rGO} / \mathrm{Co}_{0.5} \mathrm{Zn}_{0.5} \mathrm{Fe}_{2} \mathrm{O}$ 复合材料中存在双介电弛豫过程, 而 $\mathrm{rGO}$ 与 $\mathrm{Co}_{0.5} \mathrm{Zn}_{0.5} \mathrm{Fe}_{2} \mathrm{O}_{4}$ 之间的界面效应是造成双介电损耗 的原因 ${ }^{[46]}$ 。GO 是由石墨在液相中氧化和剥离而产 生的一种衍生物, 其官能团的生成会导致 GO 表面 产生大量缺陷。当 $\mathrm{GO}$ 还原为 $\mathrm{rGO}$ 时, 缺陷仍然存 在, 作为极化中心的缺陷在电磁场作用下会产生极 化弛豫, 衰减电磁波, 从而对微波的损耗产生一定 影响。从图 6 可以看到 $\mathrm{rGO} / \mathrm{Co}_{0.5} \mathrm{Zn}_{0.5} \mathrm{Fe}_{2} \mathrm{O}_{4}-3$ 的 ColeCole 半圆相对更加圆满, 因此, $\mathrm{rGO} / \mathrm{Co}_{0.5} \mathrm{Zn}_{0.5} \mathrm{Fe}_{2} \mathrm{O}_{4}-3$ 在保持现有介电弛豫特性的同时, 更好地响应了阻 抗匹配的要求。

\section{$2.6 \mathrm{rGO} / \mathrm{Co}_{0.5} \mathrm{Zn}_{0.5} \mathrm{Fe}_{2} \mathrm{O}_{4}$ 复合材料的吸波反} 射率

为了进一步揭示 $\mathrm{GO}$ 和 $\mathrm{rGO}$ 复合材料的微波 吸收特性, 基于广义传输线理论对电磁波吸收性 能进行分析。用下列公式可以计算出样品的反射系 数 $\left(R_{\mathrm{L}}\right)^{[47]}$ :

$$
\begin{gathered}
R_{\mathrm{L}}=20 \lg \left|\frac{Z_{\text {in }}-1}{Z_{\text {in }}+1}\right| \\
Z_{\text {in }}=\sqrt{\frac{\mu_{\mathrm{r}}}{\varepsilon_{\mathrm{r}}}} \tanh \left[\mathrm{j} \frac{2 \pi}{c} \sqrt{\mu_{\mathrm{r}} \varepsilon_{\mathrm{r}}} f d\right]
\end{gathered}
$$

其中, $Z_{\text {in }}$ 为输入阻抗, $c$ 为真空中的光速, $f$ 为微波频 率, $d$ 为吸收体厚度, $\varepsilon_{\mathrm{r}}$ 和 $\mu_{\mathrm{r}}$ 为材料的相对介电常数 和磁导率。图 7 给出了 $\mathrm{GO}$ 和 $\mathrm{rGO} / \mathrm{Co}_{0.5} \mathrm{Zn}_{0.5} \mathrm{Fe}_{2} \mathrm{O}_{4}$ 复合材料的 $R_{\mathrm{L}}$ 曲线。从图中可以看出 $\mathrm{GO}$ 在 $18 \mathrm{GHz}$ 处反射率为 $-2.518 \mathrm{~dB}$, 而 $\mathrm{Co}_{0.5} \mathrm{Zn}_{0.5} \mathrm{Fe}_{2} \mathrm{O}_{4}$ 在 $18 \mathrm{GHz}$ 处反射率为 $-3.451 \mathrm{~dB}$, 并且二者在低于有效吸波范 围 $\left(R_{\mathrm{L}}<-10 \mathrm{~dB}\right)$ 内没有出现吸波反射率的值。但是, $\mathrm{rGO}$ 与 $\mathrm{Co}_{0.5} \mathrm{Zn}_{0.5} \mathrm{Fe}_{2} \mathrm{O}_{4}$ 复合后, 反射率明显降低, 并 且不同比例样品在有效吸波范围内均出现了吸波反 射率的值。这不仅仅是因为 $\mathrm{GO}$ 被还原为 $\mathrm{rGO}$ 后增 强了介电损耗, 而且 $\mathrm{Co}_{0.5} \mathrm{Zn}_{0.5} \mathrm{Fe}_{2} \mathrm{O}_{4}$ 的引入增加了 复合材料的磁滞损耗并与 $\mathrm{rGO}$ 的介电损耗产生协同 效应, 增强了对电磁波的吸收。在复合材料中, $\mathrm{rGO} / \mathrm{Co}_{0.5} \mathrm{Zn}_{0.5} \mathrm{Fe}_{2} \mathrm{O}_{4}-3$ 的反射损耗在 $-10 \mathrm{~dB}$ 以下的 频率带为 $13.41 \sim 17.15 \mathrm{GHz}$, 在 $15.11 \mathrm{GHz}$ 处达到了

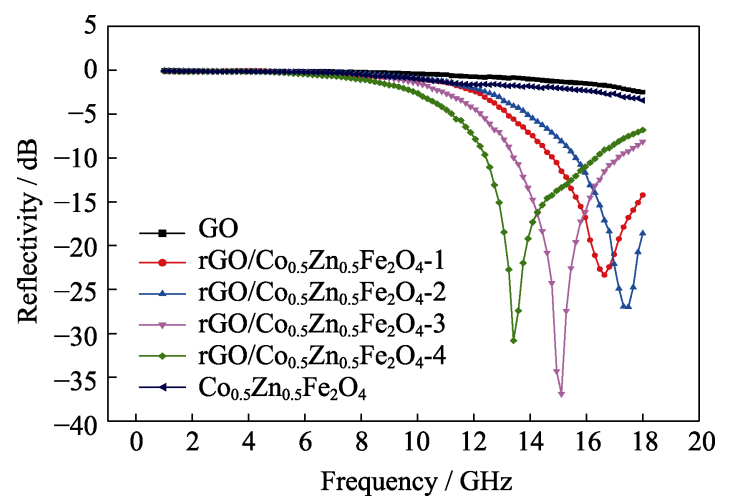

图 $7 \mathrm{GO}$ 和不同复合比例 $\mathrm{rGO} / \mathrm{Co}_{0.5} \mathrm{Zn}_{0.5} \mathrm{Fe}_{2} \mathrm{O}_{4}$ 复合材料的 反射率与曲线频率的关系曲线

Fig. 7 Curves of reflectivity and frequency of GO and $\mathrm{rGO} / \mathrm{Co}_{0.5} \mathrm{Zn}_{0.5} \mathrm{Fe}_{2} \mathrm{O}_{4}$ composites with different ratios

最小值为 $-36.89 \mathrm{~dB}$, 有效吸波频带宽为 3.74 。原因 是与反射损失相关的重要参数是阻抗匹配特性, 吸 波材料的阻抗特性应该等于自由空间损耗而实现零 反射 ${ }^{[46,48]}$ 。即两种材料复合后的阻抗匹配特性越一 致，产生协同效应越接近自由空间损耗，吸波性能 越好。而 $\mathrm{rGO} / \mathrm{Co}_{0.5} \mathrm{Zn}_{0.5} \mathrm{Fe}_{2} \mathrm{O}_{4}-3$ 的反射率损耗最小, 因而阻抗匹配特性优于其他复合比例。由此可以看 出, 吸波性能的改善来源于阻抗匹配和电磁波衰减 关键因素。 $\mathrm{Co}_{0.5} \mathrm{Zn}_{0.5} \mathrm{Fe}_{2} \mathrm{O}_{4}$ 的引入改善了磁导率的均 匀性, 提高了阻抗匹配的水平, $\mathrm{GO}$ 还原后存留的缺 陷, 可以引起电磁波多次散射和界面极化 ${ }^{[49-50]}$, $\mathrm{rGO} / \mathrm{Co}_{0.5} \mathrm{Zn}_{0.5} \mathrm{Fe}_{2} \mathrm{O}_{4}$ 复合材料的大纵横比和层状结 构会在吸收体中引起多次反射, 导致电磁波在吸收 层中传播的路径延长, 从而进一步提高 $\mathrm{rGO}$ / $\mathrm{Co}_{0.5} \mathrm{Zn}_{0.5} \mathrm{Fe}_{2} \mathrm{O}_{4}$ 复合材料的吸波能力。

\section{3 结论}

1) 通过改进 Hummers 法制备 GO, 水热法制备 $\mathrm{Co}_{0.5} \mathrm{Zn}_{0.5} \mathrm{Fe}_{2} \mathrm{O}_{4}$, 晶化法制备不同比例 $\mathrm{rGO}$ / $\mathrm{Co}_{0.5} \mathrm{Zn}_{0.5} \mathrm{Fe}_{2} \mathrm{O}_{4}$ 复合材料。

2) 在 $\mathrm{rGO} / \mathrm{Co}_{0.5} \mathrm{Zn}_{0.5} \mathrm{Fe}_{2} \mathrm{O}_{4}$ 复合材料中, 当 $\mathrm{Co}_{0.5} \mathrm{Zn}_{0.5} \mathrm{Fe}_{2} \mathrm{O}_{4}: \mathrm{GO}=1: 1$ 时, $\mathrm{Co}_{0.5} \mathrm{Zn}_{0.5} \mathrm{Fe}_{2} \mathrm{O}_{4}$ 均匀地 分散于 $\mathrm{rGO}$ 片层上, 结合性很好且没有明显的团聚。 但当 $\mathrm{Co}_{0.5} \mathrm{Zn}_{0.5} \mathrm{Fe}_{2} \mathrm{O}_{4}$ 的含量增加时, $\mathrm{Co}_{0.5} \mathrm{Zn}_{0.5} \mathrm{Fe}_{2} \mathrm{O}_{4}$ 在 $\mathrm{rGO}$ 上的分散性减弱, 出现明显的团聚现象。因 此, 当 $\mathrm{Co}_{0.5} \mathrm{Zn}_{0.5} \mathrm{Fe}_{2} \mathrm{O}_{4}$ 的含量较高时, 二者的相容 性有待进一步提高。

3) 通过对不同比例 $\mathrm{rGO} / \mathrm{Co}_{0.5} \mathrm{Zn}_{0.5} \mathrm{Fe}_{2} \mathrm{O}_{4}$ 复合材 料的复介电常数和磁导率分析, 发现 $\mathrm{rGO} /$ $\mathrm{Co}_{0.5} \mathrm{Zn}_{0.5} \mathrm{Fe}_{2} \mathrm{O}_{4}$ 复合材料的反射损耗主要是由介电 损耗产生。当 $\mathrm{Co}_{0.5} \mathrm{Zn}_{0.5} \mathrm{Fe}_{2} \mathrm{O}_{4}$ 与 $\mathrm{GO}$ 质量比为 $2: 1$ 
时制备的 $\mathrm{rGO} / \mathrm{Co}_{0.5} \mathrm{Zn}_{0.5} \mathrm{Fe}_{2} \mathrm{O}_{4}$ 复合材料的电磁损耗 性能最佳, 反射损耗在有效吸波范围 $\left(R_{\mathrm{L}}<-10 \mathrm{~dB}\right)$ 内 的频率带为 $13.41 \sim 17.15 \mathrm{GHz}$, 并且在 $15.11 \mathrm{GHz}$ 处 达到了最小值，为 $-36.89 \mathrm{~dB}$ ，有效吸波频带宽为 3.74 。

\section{参考文献:}

[1] WU G L, CHENG Y H, XIANG F, et al. Morphology controlled synthesis, characterization and microwave absorption properties of nanostructured 3D CeO 2 . Mat. Sci. Semicond. Process, 2016, 41(2): 6-11.

[2] FU W, LIU S, FAN W, et al. Hollow glass microspheres coated with $\mathrm{CoFe}_{2} \mathrm{O}_{4}$ and its microwave absorption property. J. Magn. Magn. Mater., 2007, 316(1): 54-58.

[3] HAJALILOU A , HASHIM M , MASOUDI M T. A comparative study of in-situ mechanochemically synthesized $\mathrm{Mn}_{0.5} \mathrm{Zn}_{0.5} \mathrm{Fe}_{2} \mathrm{O}_{4}$ ferrite nanoparticles in the $\mathrm{MnO} / \mathrm{ZnO} / \mathrm{Fe}_{2} \mathrm{O}_{3}$ and $\mathrm{MnO}_{2} / \mathrm{Zn} / \mathrm{Fe}_{2} \mathrm{O}_{3}$ systems. Ceramics International, 2015, 41(6): 8070-8079.

[4] ZHANG X C, WANG D P, YAO A H, et al. Optimization on preparation process of Mn-Zn ferrite powder by Sol-Gel method. Bulletin of the Chinese Ceramic Society, 2008, 27(5): 937-940.

[5] ZHAO H T, ZHANG Q, LIU R P, et al. Synthesis and magnetic properties of monodisperse $\mathrm{ZnFe}_{2} \mathrm{O}_{4}$ nanoparticles. Journal of Materials Engineering, 2016, 44(01): 103-107.

[6] LIU J, CHE R, CHEN H, et al. Microwave absorption enhancement of multifunctional composite microspheres with spinel $\mathrm{Fe}_{3} \mathrm{O}_{4}$ cores and anatase $\mathrm{TiO}_{2}$ shells. Small, 2012, 8(8): 1214-1221.

[7] GUO J, WU H, LIAO X, et al. Facile synthesis of size-controlled silver nanoparticles using plant tannin grafted collagen fiber as reductant and stabilizer for microwave absorption application in the whole Ku band. J. Phys. Chem. C, 2011, 115(48): 23688-23694.

[8] KONG L, YIN X, ZHANG Y, et al. Electromagnetic wave absorption properties of reduced graphene oxide modified by Maghemite colloidal nanoparticle clusters. J. Phys. Chem. C, 2013, 117(38): 19701-19711.

[9] SCHEDIN F, GEIM A K, MOROZOV S V, et al. Detection of individual gas molecules adsorbed on graphene. Nat. Mater., 2007, 6(9): $652-655$.

[10] ANG P K, CHEN W, WEE A, et al. Solution-gated epitaxial graphene as pH sensor. J. Am. Chem. Soc., 2008, 130(44): 14392-14393.

[11] STOLLER M D, PARK S J, ZHU Y W, et al. Graphene-based ultracapacitors. Nano Lett., 2008, 8(10): 3498-3502.

[12] EDA G, FANCHINI G, CHHOWALLA M. Large-area ultrathin films of reduced graphene oxide as a transparent and flexible electronic material. Nat. Nanotechnol., 2008, 3(5): 270-274.

[13] YOO E, KIM J, HOSONO E, et al. Large reversible Li storage of graphene nanosheet families for use in rechargeable lithium ion batteries. Nano Lett., 2008, 8(8): 2277-2282.

[14] GEIM A K. Graphene: status and prospects. Science, 2009, 324(5934): $1530-1534$

[15] BALANDIN A A, GHOSH S, BAO W Z, et al. Lausuperior thermal conductivity of single layer graphene. Nano Lett., 2008, 8(3): 902-907.

[16] LEE C, WEI X D, KYSAR J W, et al. Measurement of the elastic properties and intrinsic strength of monolayer graphene. Science, 2008, 321(5887): 385-388.

[17] LI D, MULLER M B, GILJE S, et al. Processable aqueous dispersions of graphene nanosheets. Nat. Nanotechnol., 2008, 3(2): 101-105.
[18] JUNG I, DIKIN D A, PINER R D, et al. Tunable electrical conductivity of individual graphene oxide sheets reduced at "low" temperatures. Nano Lett., 2008, 8(12): 4283-4287.

[19] CHEN H, MULLER M B, GILMORE K J, et al. Mechanically strong, electrically conductive, and biocompatible graphene paper. Adv. Mater., 2008, 20(18): 3557-3561.

[20] LIAN P C, ZHU X F, XIANG H F, et al. Enhanced cycling performance of $\mathrm{Fe}_{3} \mathrm{O}_{4}$-graphene nanocomposite as an anode material for lithium-ion batteries. Electrochim. Acta, 2010, 56(2): 834840.

[21] LI N W, ZHENG M B, CHANG X F, et al. Preparation of magnetic $\mathrm{CoFe}_{2} \mathrm{O}_{4}$-functionalized graphene sheets via a facile hydrothermal method and their adsorption properties. J. Solid State Chem., 2011, 184(4): 953-958.

[22] ZONG M, HUANG Y, ZHANG $\mathrm{N}$, et al. Influence of $(\mathrm{RGO}) /($ Ferrite) ratios and graphene reduction degree on microwave absorption properties of graphene composites. J. Alloys Compd., 2015, 644(25): 491-501.

[23] DAN C, LIU X, YU R, et al. Enhanced microwave absorption properties of flake-shaped FePCB metallic glass/graphene composites. Composites Part A, 2016, 89: 33-39.

[24] AN R, WEI H Y, HE M, et al. The progress analysis of carbonbased composites used for electromagnetic wave absorption. $\mathrm{Ma}$ terials Review, 2017, 31(21): 46-53, 61.

[25] HUMMERS W S, OFFEMAN R E. Preparation of graphitic oxide. Journal of the American Chemical Society, 1958, 80(6): 1339.

[26] KOVTYUKHOVA N I, OLLIVIER P J. Layer-by-layer assembly of ultrathin composite films from micron-sized graphite oxide sheets and polycations. Chemical Materials, 1999, 11(3): 71-78.

[27] GENG Y, WANG S J, KIM J K. Preparation of graphite nanoplatelets and graphene sheets. J. Colloid Interface Sci., 2009, 336(2): 592-598.

[28] SU J, CAO M H, REN L, et al. $\mathrm{Fe}_{3} \mathrm{O}_{4}$-graphene nanocomposites with improved Lithium storage and Magnetism properties. J. Phys. Chem. C, 2011, 115(30): 14469-14477.

[29] GABAL M A, EL-SHISHTAWY R M, ANGARI YM. Structural and magnetic properties of nano-crystalline $\mathrm{Ni}-\mathrm{Zn}$ ferrites synthesized using egg-white precursor. J. Magn. Magn. Mater., 2012, 324(14): 2258-2264.

[30] YANG H B, YE T, LIN Y, et al. Microwave absorbing properties of the ferrite composites based on graphene. J. Alloys Compd., 2016, 683: $567-574$.

[31] GRAF D, MOLITOR F, ENSSLIN K, et al. Spatially resolved raman spectroscopy of single and few-layer graphene. Nano Lett., 2007, 7(2): 238-242.

[32] BELL N J, HG Y H, DU A J, et al. Understanding the enhancement in photoelectrochemical properties of photocatalytically prepared $\mathrm{TiO}_{2}$-reduced graphene oxide composite. J. Phys. Chem. C, 2011, 115(13): 6004-6009.

[33] FU MIN, JIAO QINGZE, ZHAO YUN. Preparation of $\mathrm{NiFe}_{2} \mathrm{O}_{4}$ nanorod-graphene compoites via an ionic liquid assisted one-step hydrothermal approch and their microwave absorbing properties. Journal of Materials Chemistry A, 2013, 1(18): 5577-5586.

[34] FERRARI AC, ROBERTSON A. Interpretation of Raman spectra of disordered and amorphous carbon. J. Physiol. Rev. B, 2000, 61(20): 14095-14107.

[35] SUN XIN, HE JIANPING, LI GUOXIAN, et al. Laminated magnetic graphene with enhanced electromagnetic wave absorption properties. Journal of Materials Chemistry C, 2013, 1(4): 765-777.

[36] TUINSTRA F, KOENIG J L. Raman spectrum of graphite. $J$. Chem. Phys., 1970, 53(3): 1126-1130. 
[37] MURUGAN A V, MURALIGANTH T, MANTHIRAM A. Rapid, facile microwave-solvothermal synthesis of graphene nanosheets and their polyaniline nanocomposites for energy strorage. Chem. Mater., 2009, 22(8): 5004-5006.

[38] FU MIN, JIAO QINGZE, ZHAO YUN. In situ fabrication and characterization of cobalt ferrite nanorods/graphene composites. Materials Characterization, 2013, 86(8): 303-315.

[39] JEONG H K, LEE Y P, LAHAYE R J, et al. Evidence of graphitic AB stacking order of graphite oxides. J. Am. Chem. Soc., 2008, 130(4): 1362-1366.

[40] NETHRAVATHI C, NISHA T, RAVISHANKAR N, et al. Graphenenanocrystalline metal sulphide composites produced by a one-pot reaction starting from graphite oxide. Carbon, 2009, 47(8): 20542059.

[41] BOURLINOS A B, GOURNIS D, PETRIDIS D, et al. Graphite oxide: chemical reduction to graphite and surface modification with primary aliphatic amines and amino acids. Langmuir, 2003, 19(15): 6050-6055.

[42] ZHANG X F, DONG X L, HUANG H, et al. Microwave absorption properties of the carbon-coated nickel nanocapsules. Appl. Phys. Lett., 2006, 89(5): 053115-1-3.

[43] TIANJIAO B, YAN Z, XIAOFENG S, et al. A study of the electromagnetic properties of cobalt-multiwalled carbon nano- tubes(co-mwcnts)composites. Materials Science And Engineering: $B, 2011$, 176(12): 906-912.

[44] HASSAN A, KHAN M A, ASGHAR M, et al. Nanocrystalline $\mathrm{Zn}_{1-x} \mathrm{Co}_{0.5 x} \mathrm{Ni}_{0.5 x} \mathrm{Fe}_{2} \mathrm{O}_{4}$ ferrites: fabrication via co-precipitation route with enhanced magnetic and electrical properties. J. Magn. Magn. Mater, 2015, 393: 56-77.

[45] FRENKEL J, DORFMAN J. Spontaneous and induced magnetisation in ferromagnetic bodies. Nature, 1930, 126(3173): 274-275.

[46] YANG HAIBO, YE TING, LIN YING, et al. Microwave absorbing properties of the ferrite composites based on grapheme. J. Alloys Compd., 2016, 683: 567-574.

[47] MILES P A, WESTPHAL W B, VON HIPPEL A. Dielectric spectroscopy of ferromagnetic semiconductors rev. Mod. Phys., 1957, 29(3): 279-307.

[48] MA Z, WANG J B, LIU Q F, et al. Microwave absorption of electroless Ni-Co-P-coated $\mathrm{SiO}_{2}$ powder. Appl. Surf. Sci., 2009, 255(13): 6629-6633.

[49] RUTTER G M, CRAIN J N, GUISINGER N P, et al. Scattering and interference in epitaxial graphene. Science, 2007, 317(5835): 219-222.

[50] SUN S L, HE Q, XIAO S Y, et al. Gradient-index meta-surfaces as a bridge linking propagating waves and surface waves. Nat. Mater., 2012, 11(5): 426-431. 